Artigo

\title{
Análise sinótica e Termodinâmica dos Eventos com Trovoadas para o Estado de Alagoas no Período de 15 Anos 1998-2012
}

\author{
Edwans Silva Cordeiro ${ }^{1}$, Natalia Fedorova ${ }^{1}$, Vladimir Levit ${ }^{1}$ \\ ${ }^{1}$ Instituto de Ciências Atmosféricas, Universidade Federal de Alagoas, Maceió, AL, Brasil.
}

Recebido em: 9 de Junho de 2016 - Aceito em: 10 de Março de 2018

\begin{abstract}
Resumo
Alagoas é afetado pelas trovoadas e buscando mitigar tais transtornos, este trabalho propõe-se a compreender melhor os sistemas sinóticos associados às mesmas. Dados do Departamento do Controle do Espaço Aéreo (DECEA) e do satélite Tropical Rainfall Measuring Mission (TRMM) foram utilizados para estimar a frequência das trovoadas. Também foram aplicados dados de reanálise do National Centers for Environmental Prediction (NCEP). Foram encontrados no Estado cerca de 212 eventos com trovoadas em 15 anos. A maior ocorrência foi observada em 2008 (21 casos) e a menor ocorrência em 2012 (04 casos). Os meses com maior frequência de trovoadas foram Janeiro, Fevereiro e Março. $\mathrm{Na}$ identificação dos sistemas sinóticos foram encontrados: Cavados em Altos Níveis, Zona Frontal, Zona de Convergência Intertropical (ZCIT), Vórtice Ciclônico de Altos Níveis (VCAN) e Vórtice Ciclônico de Médios Níveis (VCMN). Os sistemas que mais apresentaram casos com trovoadas foram VCAN e Cavados em Altos Níveis. Os Índices de Instabilidade mostraram forte instabilidade para o VCAN, ZCIT e Cavados em Altos Níveis e fraca instabilidade para o VCMN.
\end{abstract}

Palavras-chave: trovoadas, sistemas sinóticos, índices de instabilidade.

\section{Synoptic and Thermodynamic Analysis of Events with Thunderstorms for}

\section{Alagoas State in a Period of 15 Years 1998-2012}

\begin{abstract}
The Brazilian state of Alagoas is occasionally affected by thunderstorms. Aiming to mitigate these disorders, the present study suggests a better understanding of synoptic systems associated with thunderstorms. DECEA and TRMM satellite data were used to estimate the frequency of thunderstorms. Reanalysis data from NCEP have been also used. Thereby, 212 thunderstorm events were found during 15 years. The highest thunderstorm frequency ( 21 events) was observed in 2008 and the smallest frequency was observed in 2012 (04 events). January, February and March are the months with the highest thunderstorms number. Five synoptic systems associated with thunderstorms have been identified: Trough at the Upper Levels (TUL), Intertropical Convergence Zone (ITCZ), Upper Tropospheric Cyclone Vortex (UTCV), Frontal Zone (FZ) and Middle Tropospheric Cyclone Vortex (MTCV). UTCV and TUL were detected as the systems with the highest thunderstorms number. The Instability Indexes showed strong instability for the UTCV, ITCZ and TUL, and low instability for the MTCV.
\end{abstract}

Keywords: Thunderstorm, synoptic systems, instability index.

\section{Introdução}

Trovoada é utilizada como um "termo genérico" para indicar a existência de trovões (Brito, 2011), sendo ainda conseqüência de processos que existem dentro de células convectivas (Felício, 2005), ou seja, associada à presença de nuvens do tipo Cumulonimbus. Outra definição é observada em Vianello e Alves (2000), o qual define trovoada como uma ou várias descargas bruscas de eletricidade atmosférica, manifestando-se por uma claridade breve e intensa (relâmpago) e por um ruído seco (trovão).

\footnotetext{
${ }^{1}$ Autor de correspondência: Edwans Silva Cordeiro, divascordeiro@gmail.com.
} 
Para que um fenômeno meteorológico deste porte possa ocorrer, é necessária a presença de Sistemas Sinóticos. Estes sistemas são considerados de escala sinótica e podem estar associados a altos índices de precipitação (Silva, 2011).

Dentre os sistemas sinóticos mais comuns, destacam-se neste trabalho:

- Cavados Báricos, que são ondas tropicais ou máximas curvaturas ciclônica dos ventos alísios (Simpson, 1968), que atuam no Nordeste brasileiro (NEB) durante qualquer época do ano (Rodrigues, et al. 2008). Em Alagoas, estes estão associados aos diferentes tipos de fenômenos meteorológicos, a exemplo das trovoadas.

- Zona de Convergência Intertropical (ZCIT), que é um sistema facilmente visualizado em imagens de satélite como uma banda de nuvens convectivas que se estende em uma faixa ao longo da região equatorial (Cavalcanti, et al. 2009). É o fator mais importante na determinação de quão abundante ou deficiente serão as chuvas no setor norte do NEB.

- Vórtices Ciclônicos em Altos Níveis(VCAN) são sistemas caracterizados por centros de baixa pressão que se originam na alta troposfera, podendo se estender até níveis médios, dependendo da instabilidade atmosférica (Cavalcanti, et al. 2009). O tempo de vida desses sistemas varia, em média, entre 7 a 10 dias (Fedorova, 2011).

- Vórtices Ciclônicos em Médios Níveis(VCMN) consistem em um sistema de vórtices o qual apresenta circulação ciclônica fechada somente em médios níveis, ou seja, entre $700-400 \mathrm{hPa}$ (Fedorova et al. 2016). Segundo Silva et al. (2011) e Santos (2012), as maiores frequências de VCMN foram observadas no verão. Este sistema também pode estar fortemente ligado à ocorrência de fenômenos adversos e, por isso, já é bastante estudado por Fedorova et al. (2015) o que, posteriormente, foi publicado sua real influência no tempo em Alagoas.

- Sistemas Frontais que, segundo Cavalcanti, et al. (2009), durante o verão, frequentemente se posicionam ao longo da costa do Brasil, chegando a atingir o NEB, originando períodos prolongados de chuva forte. Fedorova et al. (2015), em seu estudo mais recente, descreveu novos métodos de identificação das frentes nas regiões tropicais, bem como sua influência no tempo do NEB.

Quanto à ocorrência das trovoadas para o NEB, há certa carência de estudos voltados a este tema, em especial para o Estado de Alagoas. Entretanto, alguns estudos anteriores, voltados às precipitações intensas e trovoadas no NEB (Brito, 2011; Silva et al. 2011), já foram elaborados, destacando a frequência e análise de casos com trovoadas para Alagoas.

A influência destes sistemas sinóticos se tornou importante em relação às trovoadas. Este trabalho tem como objetivo principal fazer a análise da frequência dos casos de trovoadas entre os anos de 1998 a 2012, dando seguimento ao estudo previamente desenvolvido por Brito (2008). Por conseguinte, serão apresentadas as análises sinóticas de alguns eventos encontrados nos anos incorporados ao estudo. A análise termodinâmica também foi aplicada neste trabalho, através dos índices de instabilidade. Com isso, foi possível fazer a relação entre a intensidade do fenômeno meteorológico e o sistema sinótico atuante.

\section{Material e Métodos}

\section{1 Área em estudo}

De acordo com a classificação de Köppen, toda a metade oriental do Estado possui clima tropical e quente, com precipitação pluviométrica de outono/inverno, entre $1.000 \mathrm{~mm}$ a $1.500 \mathrm{~mm}$. Porém, parte do leste alagoano, próximo à divisa com o Estado de Pernambuco, possui clima tropical com chuvas de outono a inverno e médias pluviométricas anuais entre $1.500 \mathrm{~mm}$ a $2.200 \mathrm{~mm}$. A metade ocidental do Estado, que corresponde ao agreste e sertão, apresenta condições semiáridas, com clima seco e quente, com precipitação pluviométrica média anual no sertão entre $400 \mathrm{~mm}$ a $600 \mathrm{~mm}$ e no agreste de $600 \mathrm{~mm}$ a $900 \mathrm{~mm}$ (Barros et al. 2012).

A cidade de Maceió, por sua vez, possui um regime pluviométrico bem distribuído ao longo do ano, sendo mais frequentes dentre os meses de outono/inverno, como mostra a Fig. 1.

\subsection{Levantamento dos eventos com trovoadas}

\subsubsection{Dados do Aeroporto Zumbi dos Palmares}

O levantamento do número de casos com trovoadas e suas respectivas datas, foi realizado através do banco de dados do Aeroporto Internacional Zumbi dos Palmares, que está localizado na cidade de Maceió, Alagoas. Esses dados possuem alcance máximo de $20 \mathrm{~km}$ de raio do Aeroporto e foram obtidos do Departamento de Controle do Espaço Aéreo (DECEA), sendo observados e registrados através do código SYNOP. Vale salientar que, estes dados são fornecidos de forma horária, logo, foi contada como evento com trovoada, a atuação do fenômeno meteorológico indicado pelo símbolo (『).

\subsubsection{Dados do satélite TRMM}

O satélite Tropical Rainfall Measuring Mission (TRMM), é particularmente dedicado à identificação de chuvas em regiões tropicais e subtropicais da Terra. O satélite possui 5 sensores, porém o mais utilizado para verificar a frequência das descargas atmosféricas foi o Lightning Imaging Sensor (LIS).

O sensor LIS, a bordo do satélite TRMM, é um instrumento usado para detectar a distribuição e a variabilidade das descargas atmosféricas que ocorrem nas regiões 


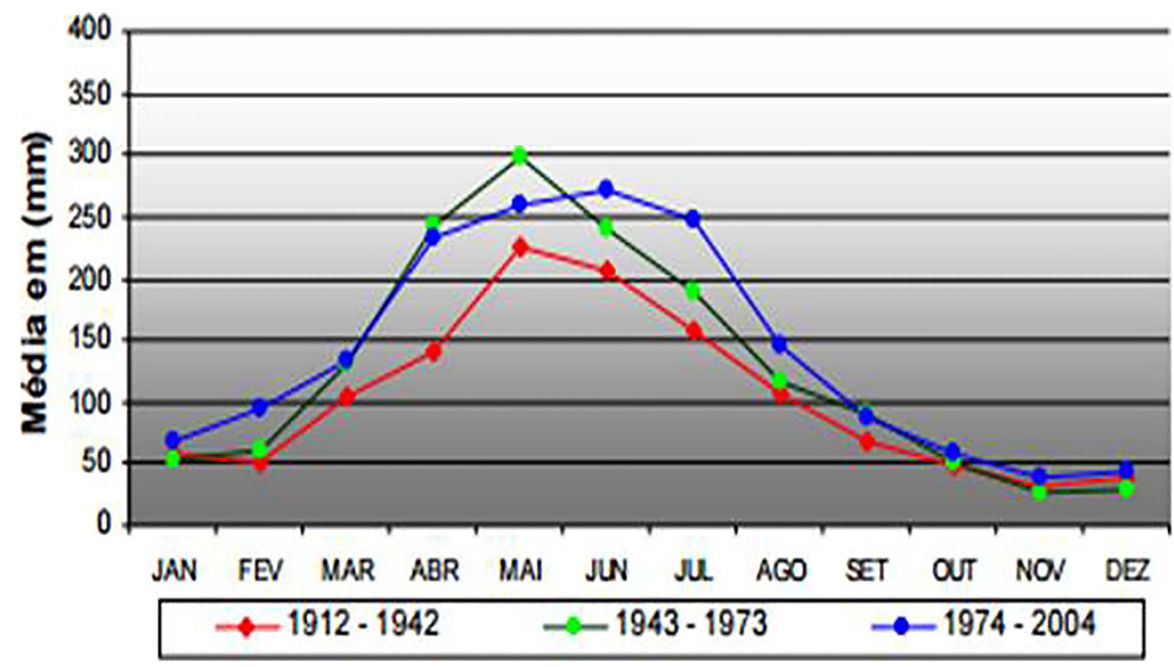

Fig. 1 - Normais climatológicas da precipitação em Maceió/AL em três períodos distintos. Fonte: SEMARH/AL.

tropicais do globo. Este sensor é constituído por uma câmera otimizada com resolução em escala de 4 a $7 \mathrm{~km}$ sobre uma grande região $(600$ x $600 \mathrm{~km})$ da superfície da Terra. $\mathrm{O}$ instrumento registra o tempo de ocorrência, mede a energia radiante e determina a localização de eventos de raios dentro de seu campo de visão (Brito, 2011).

O levantamento dos dados feito pelo TRMM e pelo DECEA auxiliou numa melhor identificação do fenômeno meteorológico em estudo, uma vez que, houve casos que foram registrados, por exemplo, pelo DECEA e que não foram registrados pelo TRMM (e vice-versa). Desta forma, foi importante o estudo de ambas as fontes, a fim de conseguir resultados mais precisos sobre as trovoadas.

\subsection{Dados de reanálise do National Centers for Environmental Prediction do National Center for Atmospheric Research (NCEP/NCAR)}

O Projeto Reanálise NCEP/NCAR, é um projeto em conjunto com o National Centers for Environmental Prediction (NCEP, ex-"NMC") e do National Center for Atmospheric Research (NCAR). O NCEP/NCAR utiliza um sistema de análise/previsão e realiza a assimilação de dados usando-os desde 1948 até o presente. O grande subconjunto destes dados está disponível a partir do Physical Sciences Division (PSD) em seu formato original diário. Os dados são exportados diariamente em horários disponíveis em $0000 \mathrm{Z}, 0600 \mathrm{Z}, 1200 \mathrm{Z}$ e $1800 \mathrm{Z}$.

Os dados foram utilizados para construção de diversos campos a exemplo de linhas de corrente, nos três níveis principais da atmosfera, sendo eles: 1000 (baixos), 500 (médios) e 300 (altos) hPa para uma área que compreende $20^{\circ} \mathrm{N} ; 50^{\circ} \mathrm{S}$ de latitude e $80^{\circ} \mathrm{W} ; 20^{\circ} \mathrm{W}$ de longitude, todos a cada 6 horas, com uma resolução de $2,5^{\circ} \mathrm{x}$ $2,5^{\circ}$. O Aeroporto Zumbi dos Palmares (AZP) está localizado dentro dessa área.

\subsection{Identificação dos sistemas}

Como parte principal deste trabalho, fez-se necessária a análise dos diferentes campos sinóticos para identificação de sistemas que compõem a atmosfera no momento da trovoada. É importante frisar que, nem todos os campos sinóticos foram mostrados neste trabalho, porém, para análise e identificação dos sistemas, foi imprescindível o estudo dos mesmos. O principal campo sinótico utilizado foi Linha de Corrente (importante na identificação dos sistemas sinóticos). Os horários para verificação do sistema são àqueles adotados pelo NCEP. Os mapas foram plotados levando em consideração os três níveis principais da atmosfera: Baixos, Médios e Altos Níveis (ver item 2.3).

Segundo Silva et al. (2011), a construção desses mapas, em cada nível, facilita a identificação dos sistemas sinóticos e de mesoescala associados aos casos com trovoadas em Alagoas, uma vez que através da circulação exposta neles, pode-se notar a atuação de cavados (cristas), ciclones (anticiclones) e a confluência (difluência) dos ventos em cada nível.

Sistemas como Cavados, Cristas, Circulações ciclônicas e anticiclônicas foram de fácil identificação, pois puderam ser notados em quaisquer níveis atmosféricos. Entretanto, para sistemas como ZCIT, VCAN e VCMN, houve um limite de observação, pois se tratam de sistemas sinóticos que ocorrem somente em uma determinada camada da atmosfera.

Para análise do VCAN, foram considerados campos de linhas de corrente desde $500 \mathrm{hPa}$ até $300 \mathrm{hPa}$. A princípio o VCAN é observado somente em altos níveis (até $200 \mathrm{hPa}$ ), porém quando há casos mais intensos o mesmo pode se estender até $500 \mathrm{hPa}$. A ZCIT também foi observada nas Linhas de Corrente e magnitude do vento. Como já se sabe a ZCIT só pode ser vista em níveis baixos da atmosfera, logo, as análises associadas a este sistema 
foram elaboradas somente em $1000 \mathrm{hPa}$. Por fim, também foi analisado um sistema considerado pouco estudado na literatura, mas que influencia nas precipitações e trovoadas do Estado. Trata-se do VCMN identificado nas imagens de Linhas de Corrente. O que diferencia este sistema do VCAN é o fato do mesmo estar presente somente em médios níveis, ou seja, desde $700 \mathrm{hPa}$ até cerca de $400 \mathrm{hPa}$. Ressalta-se que o VCMN não é observado em baixos e altos níveis.

\subsection{Análise termodinâmica}

A análise termodinâmica da atmosfera, associada à formação das trovoadas, foi realizada através de Perfis Verticais de Reanálise para a cidade de Maceió. Estes perfis foram construídos em um ponto de grade que foi escolhido dentro do Estado de Alagoas em 9, $6^{\circ} \mathrm{S}$ de latitude e $36^{\circ} \mathrm{W}$ de longitude, através dos dados do NCEP/NCAR, onde a partir daí foram plotados pelo software GrADS em 10 níveis diferentes $(1000,950,900,850,800,700,600$, 500,400 e $300 \mathrm{hPa}$ ). Ressalta-se ainda que, estes perfis foram elaborados por falta de dados de radiossondagem para o Estado de Alagoas. Tais perfis contribuíram principalmente na identificação da instabilidade e umidade da atmosfera, além de apontar valores numéricos dos índices de instabilidade, descritos a seguir.

Para verificar a capacidade potencial da atmosfera no desenvolvimento de tempestades, foram avaliados a partir de Perfis de Reanálise os seguintes Índices de Instabilidade que, segundo Cordeiro (2011), em seu estudo preliminar, mostrou que tais índices podem fornecer informações úteis na análise da instabilidade térmica da atmosfera.

\subsection{1 Índice de Levantamento (Lifted Index - LI)}

$\mathrm{O}$ índice de instabilidade por levantamento é definido por Galway (1956) pela Eq. (1): LI = T500 - T500' onde, T500 é a temperatura do ar ambiente em $500 \mathrm{hPa}$ e; T500' é a temperatura de uma parcela de ar (ambas em graus Celsius), após ascender do nível de $850 \mathrm{hPa}$ até $500 \mathrm{hPa}$ (considerando saturação).

Valores negativos de LI indicam condições instáveis, por exemplo, a parcela de ar ascendente é flutuante em níveis médios. O índice LI é uma informação importante para analisar o grau de instabilidade atmosférica, especialmente quando combinada com o CAPE.

A Tabela 1 apresenta os valores críticos do Índice LI.

\subsubsection{CAPE}

Segundo Loureiro (2005), o valor de CAPE, também conhecido como o trabalho associado ao empuxo, corresponde à área entre a curva de temperatura do ambiente (dada pela sondagem) e a curva da adiabática saturada, do Nível de Convecção Espontânea (NCE) até o Nível de Equilíbrio (NE).
Tabela 1 - Índice LI. (Fonte: http://www.srh.noaa.gov/ffc/html/gloss2. shtml)

\begin{tabular}{|c|c|}
\hline Índice LI & Potencial de ocorrência de atividade convectiva \\
\hline $\mathrm{LI}>2$ & Sem atividade convectiva significante \\
\hline $0<\mathrm{LI}<2$ & Possibilidade de ocorrência de convecção rasa \\
\hline$-2<\mathrm{LI}<0$ & Possibilidade de ocorrência de convecção profunda \\
\hline$-4<\mathrm{LI}<-2$ & $\begin{array}{l}\text { Possibilidade de chuvas e trovoadas (porém, pouco } \\
\text { severas) }\end{array}$ \\
\hline $\mathrm{LI}<-4$ & Possibilidade de chuvas torrenciais com trovoadas severas \\
\hline
\end{tabular}

Quanto maior o CAPE, maior a velocidade do ar a subir, o que significa maior intensidade da trovoada. $\mathrm{O}$ valor do CAPE é utilizado na verificação das condições de instabilidade da atmosfera provocada por processos convectivos.

O valor do CAPE pode ser calculado a partir da Eq. (2):

$$
C A P E=\int_{z=N C E}^{z=N E} g \frac{\Delta \theta_{e}}{\theta_{e s}} d Z
$$

Para a qual NE é nível de equilíbrio (térmico) [m]; NCE é nível de convecção espontânea [m]; g é aceleração da gravidade $[\mathrm{m} / \mathrm{s} 2] ; \Delta \Theta_{e}$ é diferença entre a temperatura potencial equivalente da parcela em superfície e a temperatura potencial equivalente saturada do ambiente, em cada nível $\left[{ }^{\circ} \mathrm{C}\right]$, dado pela equação:

$$
\Delta \theta_{e}=\theta_{e p}-\theta_{e s}
$$

$\Theta_{e p}$ é a temperatura potencial equivalente da parcela em superfície $\left[{ }^{\circ} \mathrm{C}\right] ; \Theta_{e s}$ é a temperatura potencial equivalente saturada do ambiente, dada pela sondagem $\left[{ }^{\circ} \mathrm{C}\right]$.

A Tabela 2 apresenta os valores limites de CAPE, indicando o potencial para o desenvolvimento de tempestades (Duarte et al. 2002):

Embora esses índices de instabilidade tenham sido originalmente concebidos para as latitudes médias do Hemisfério Norte, eles podem ser úteis para a previsão de sistemas convectivos no Brasil, em particular para a região sul do país (Hallack e Pereira Filho, 2012). Para as regiões tropicais do país existe a necessidade de utilizá-los como base conceitual.

Tabela 2 - Índice CAPE. (Fonte: Duarte et al. 2002).

\begin{tabular}{ll}
\hline CAPE & Potencial de convecção \\
\hline$<300$ & Baixa ou nenhuma \\
$300-1000$ & Fraca \\
$1000-2500$ & Moderada \\
$2500-3000$ & Forte \\
\hline
\end{tabular}




\section{Resultados}

\subsection{Estudo da frequência das trovoadas}

A frequência dos dias com trovoadas, observada tanto pelo sensor LIS quanto pelos dados do DECEA, registrou 212 dias com trovoadas. A Tabela 3 e a Fig. 2 indicam os meses com maior presença da trovoada em Alagoas, onde os meses mais predominantes foram Janeiro, Fevereiro e Março apresentando 44, 40 e 43 casos, respectivamente. Entretanto, meses em que predominam o inverno e a primavera (de Junho a Novembro), a frequência foi menor, especialmente em Setembro, quando não se obteve registro.

Em comparação a Fig. 1, observa-se que o período de maior ocorrência das trovoadas é inversamente proporcional ao período de maior ocorrência das precipitações. Tal situação pode ser explicada pela presença de sistemas meteorológicos que atuam durante esta época do ano (final de primavera, verão e início de outono), deixando a atmosfera instável e, consequentemente, facilitando a formação de nuvens de grande desenvolvimento vertical. Já durante o inverno, a atmosfera apresenta-se fria e seca na maior parte do período, com maior predomínio de nuvens stratiformes. Com isso, as chuvas são mais frequentes e com pouca ou nenhuma trovoada.

A Tabela 4 indica os valores da soma anual dos dias com trovoadas registrados pelo satélite TRMM com os registrados pelo DECEA e, também, a classificação dos ENOS para cada ano.

Na Tabela 4 nota-se que o ano de 2008 apresentou o maior número de casos dentre os anos estudados, somando de 21 casos por ano. Porém, não foi possível observar o mesmo para o ano de 2012, pois o mesmo obteve um decréscimo acentuado no número de eventos registrados, identificando apenas 04 (quatro) casos, valor este considerado um dos menores desde 1998, quando apresentou 06 (seis) casos.

Estas trovoadas também estiveram diretamente ligadas aos ENOS, sendo observada maior ocorrência de eventos com trovoadas em anos de La Niña (a exemplo de 2007, 2008 e 2010) e diminuição destes em anos que apresentaram condições de normalidade e El Niño (a exemplo de 1998, 2009 e 2012). É importante ressaltar que, como a fase do ENOS normalmente não dura um ano inteiro, para classificação do mesmo foi considerada a fase com maior atuação durante $o$ ano.
Tabela 3 - Frequência e soma de trovoadas em um período de 15 anos (1998-2012), utilizando dados do Aeroporto Zumbi dos Palmares e do Satélite TRMM.

\begin{tabular}{|c|c|c|c|c|c|c|c|c|c|c|c|c|c|}
\hline Ano/Mês & $\mathrm{J}$ & $\mathrm{F}$ & M & A & M & $\mathrm{J}$ & $\mathrm{J}$ & A & $\mathrm{S}$ & $\mathrm{O}$ & $\mathrm{N}$ & $\mathrm{D}$ & Soma/Ano \\
\hline 1998 & 3 & & 2 & 1 & & & & & & & & & 6 \\
\hline 1999 & 1 & 1 & 4 & & 2 & & & & & & 1 & 4 & 13 \\
\hline 2000 & 5 & 3 & & & 2 & & & & & & 1 & & 11 \\
\hline 2001 & & & 2 & & & & 1 & & & 2 & & 7 & 12 \\
\hline 2002 & 4 & 4 & 2 & 2 & & & & & & & 3 & 1 & 16 \\
\hline 2003 & 4 & 5 & 2 & 2 & & & & 2 & & & & 1 & 16 \\
\hline 2004 & 5 & 2 & 2 & & 1 & & & & & & 1 & 1 & 12 \\
\hline 2005 & 4 & 2 & 5 & 1 & 1 & 1 & & & & & & 3 & 17 \\
\hline 2006 & & 4 & 5 & 6 & 2 & & & & & & 2 & & 19 \\
\hline 2007 & 1 & 8 & 3 & 6 & 1 & & & & & & & 1 & 20 \\
\hline 2008 & 2 & 5 & 8 & 1 & 3 & & & & & & & 2 & 21 \\
\hline 2009 & & 1 & 1 & 2 & 3 & & & & & & & 2 & 9 \\
\hline 2010 & 7 & 1 & 4 & 3 & & & & & & 3 & & 2 & 20 \\
\hline 2011 & 5 & 3 & 3 & 4 & 1 & & & & & & & & 16 \\
\hline 2012 & 3 & 1 & & & & & & & & & & & 4 \\
\hline Soma/Mês & 44 & 40 & 43 & 28 & 16 & 2 & 1 & 2 & 0 & 5 & 8 & 24 & 212 casos \\
\hline
\end{tabular}

\section{$\mathbf{N}^{\circ}$ de casos mensais em 15 anos}

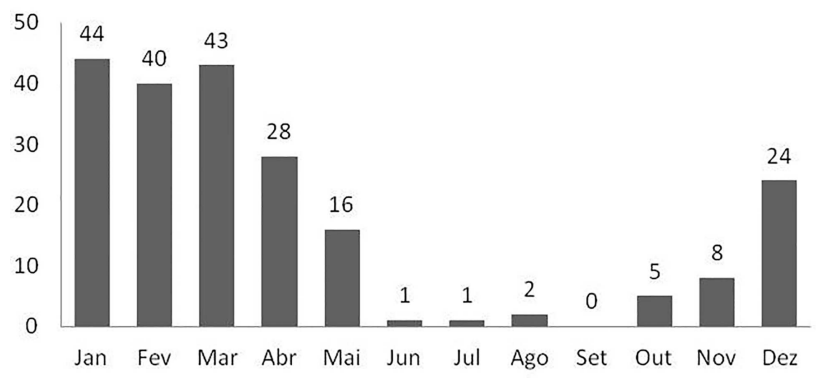

Fig. 2 - Número de eventos com trovoadas ocorrentes em todos os meses, dentre o período de 15 anos (1998-2012).

\subsection{Sistemas sinóticos associados às trovoadas}

Foi feita análise dos sistemas sinóticos que atuaram de forma direta no desenvolvimento da trovoada em Alagoas. Alguns sistemas típicos da atmosfera foram encontrados dentre os dias analisados e, além disso, também foi observado o posicionamento da trovoada em relação ao sistema sinótico.

Para identificar o sistema atuante e seu posicionamento, foi feito um levantamento dos horários em que ocorreu a trovoada. Importante frisar que nem todos os

Tabela 4 - Soma anual dos dias com trovoadas registrados pelo satélite TRMM com os registrados pelo DECEA e classificação dos ENOS para cada ano, sendo EN - El Niño; LN - La Niña; N - Condição de Neutralidade. Fonte: TRMM, DECEA, CPTEC, NOAA e Bureau of Meteorology.

\begin{tabular}{|c|c|c|c|c|c|c|c|c|c|c|c|c|c|c|c|}
\hline Ano & 98 & 99 & 00 & 01 & 02 & 03 & 04 & 05 & 06 & 07 & 08 & 09 & 10 & 11 & 12 \\
\hline Soma & 6 & 13 & 11 & 12 & 16 & 16 & 12 & 17 & 19 & 20 & 21 & 9 & 20 & 16 & 4 \\
\hline ENOS & EN & LN & LN & LN & EN & $\mathrm{N}$ & $\mathrm{N}$ & $\mathrm{N}$ & $\mathrm{N}$ & LN & LN & $\mathrm{N}$ & LN & LN & $\mathrm{N}$ \\
\hline
\end{tabular}


eventos apresentaram horários definidos, devido aos erros constantes durante o carregamento das imagens do sensor LIS. Portanto, pode-se dizer que, em um total de 212 casos, 161 possuíram horário da trovoada e 51 não possuíram. Deste modo, todos os 161 dias foram detalhadamente analisados. Os sistemas sinóticos encontrados foram:

- Cavado em Altos Níveis

- VCAN

- Sistemas Frontais

- ZCIT

- $\mathrm{VCMN}$

Posteriormente foi encontrado o número de eventos para cada sistema sinótico. A Fig. 3 abaixo apresentou maior número de casos com trovoadas na presença de Cavado em Altos Níveis com 40 casos e VCAN com 94 casos; sistemas estes que ocorrem com frequência em casos com trovoadas, nos meses acima citados.

Outros sistemas também foram visualizados durante a presença do fenômeno, porém apresentando poucos casos. São eles, ZCIT com 15 casos; VCMN com apenas 02 casos; Zona Frontal apontando 10 casos.

\section{$\mathbf{N}^{\circ}$ de casos por Sistema Sinótico}

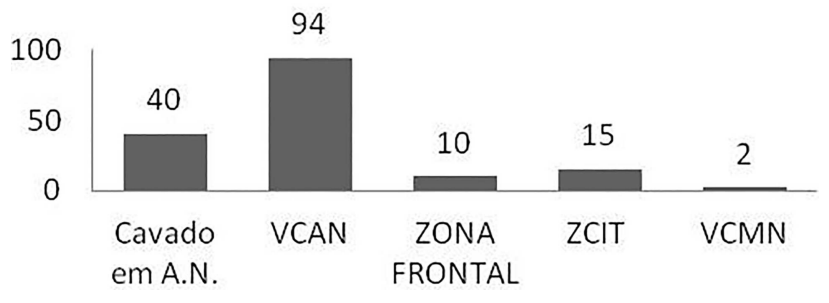

Fig. 3 - Número de eventos com trovoadas encontrados para cada sistema sinótico.

A seguir, a Fig. 4 apresenta exemplos de eventos com trovoada com cada um dos cinco sistemas sinóticos.

\subsection{Posicionamento das trovoadas nos sistemas sinóticos}

O posicionamento da trovoada foi verificado em relação ao sistema sinótico atuante. Para isto, foram consideradas duas configurações: (1) para sistema de

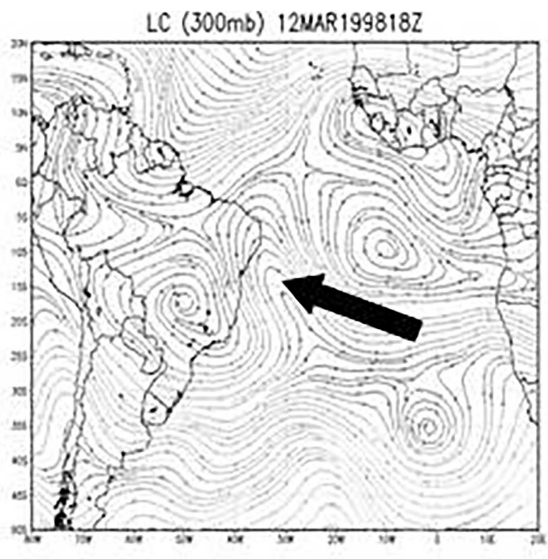

(a)

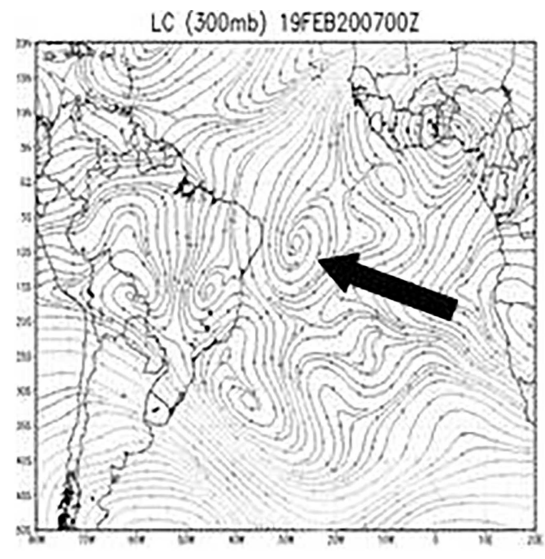

(b)

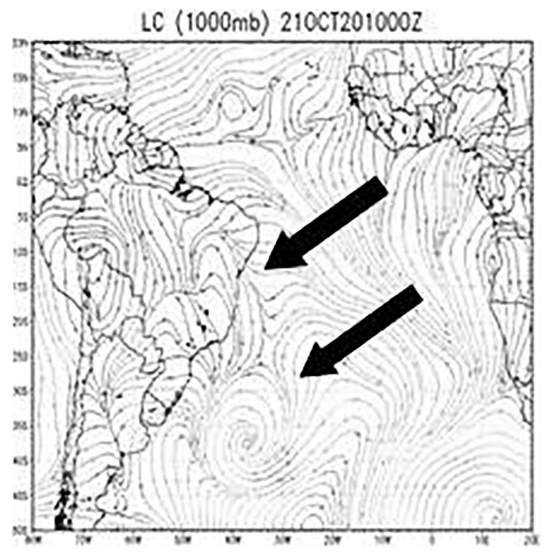

(c)

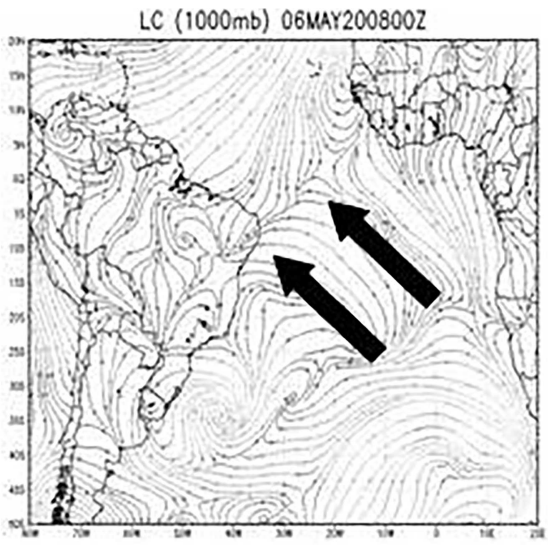

(d)

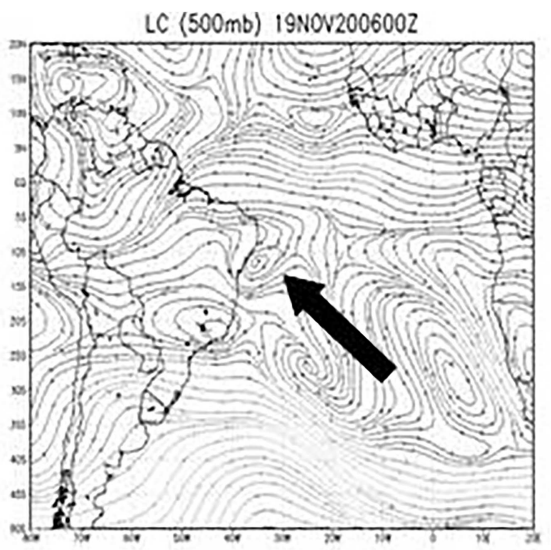

(e)

Fig. 4 - Exemplo de sistemas sinóticos encontrados nos dias com trovoadas, indicadas pelas setas: Cavado em Altos Níveis (a); VCAN (b); Sistema Frontal (c); ZCIT (d); VCMN (e). 
circulação aberta, especialmente, para Cavado em Altos Níveis e; (2) para sistemas de circulação fechada como os Vórtices Ciclônicos.

A Fig. 5 mostrou que, para Cavado em Altos Níveis, a trovoada localizou-se com maior predominância entre a

\section{Cavado em Altos Níveis}

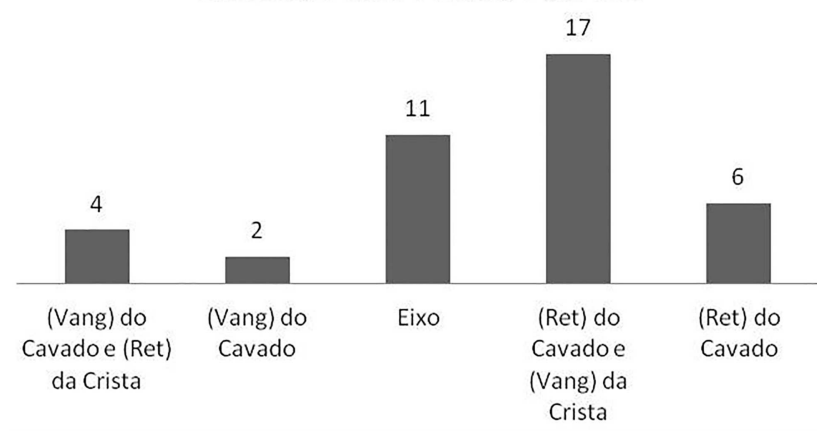

Fig. 5 - Número de eventos com trovoadas referentes ao posicionamento de seus sistemas sinóticos com circulação aberta.
Retaguarda do Cavado e Vanguarda da Crista, apresentando 17 casos ao total (Fig. 5c). Para eventos onde mostra a trovoada à Retaguarda do Cavado, considerado sistema intenso, obteve-se 6 casos (Fig. 5d). Por outro lado, a menor predominância de trovoadas ocorreu na Vanguarda do referido sistema, apontando apenas 02 casos (Fig. 5b). Trovoadas localizadas entre a Vanguarda do Cavado e Retaguarda da Crista foram encontradas em apenas 04 casos (Fig. 5a). Por fim, 11 eventos indicaram trovoadas posicionadas em seu Eixo (Fig. 5e).

A Fig. 6 apresenta exemplos de configurações de eventos com trovoada para o Cavado em Altos Níveis.

Os sistemas sinóticos de circulação fechada, apresentados na Fig. 7, apontaram uma grande distribuição das trovoadas em todas as regiões do VCAN, sendo sua maior predominância observada a Noroeste com 15 casos, Norte e Nordeste com 14 casos, ou seja, quando o VCAN atua mais sobre o Oceano ou em latitudes mais altas (próximo aos subtrópicos). Em seguida, foram identificados casos a Oeste e Sudoeste do VCAN, ambos apresentando 13 casos. Ainda no mesmo sistema, a trovoada foi vista a

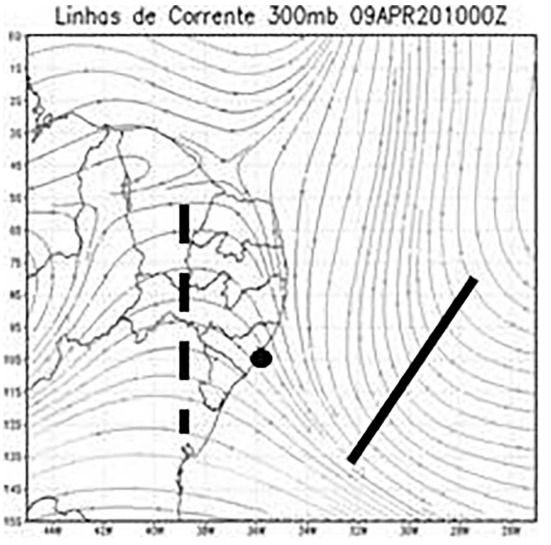

(a)

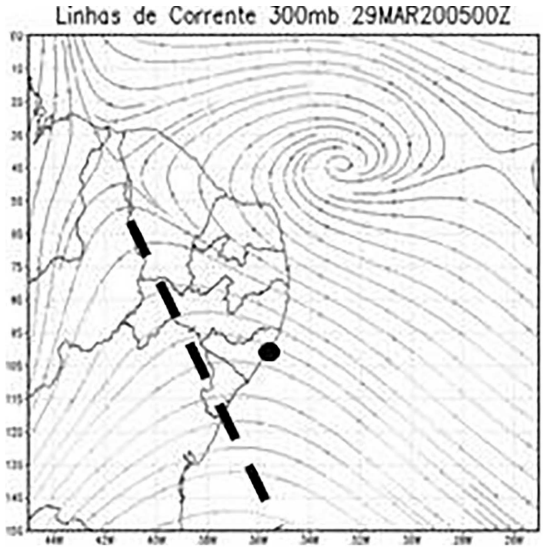

(b)

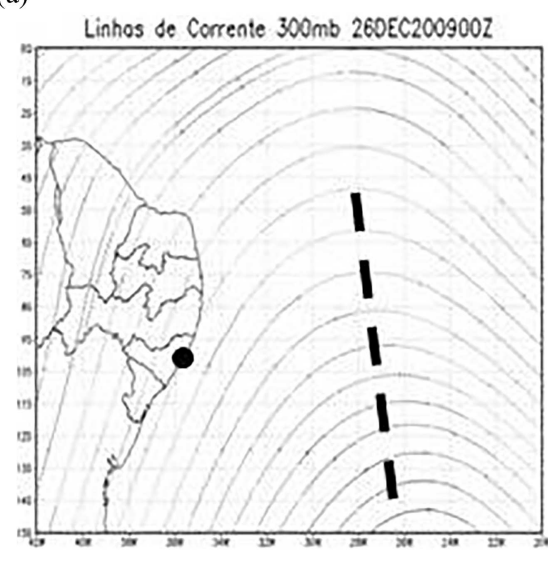

(d)

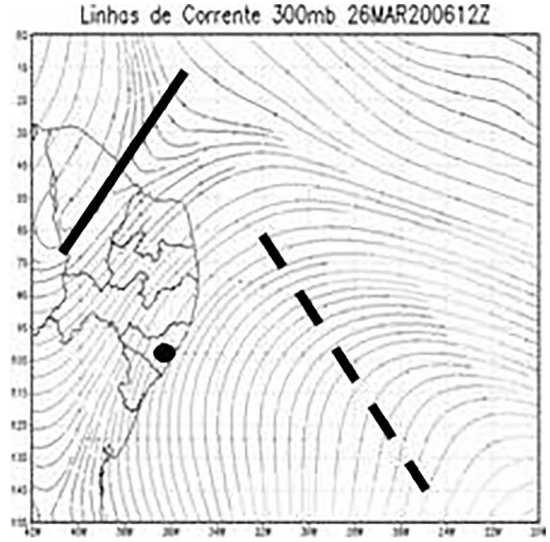

(c)

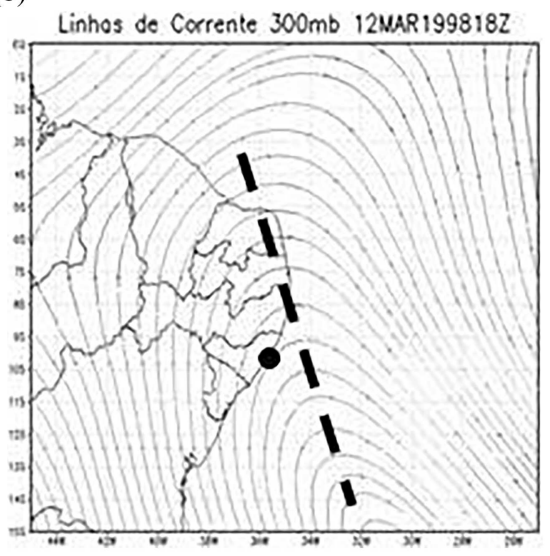

(e)

Fig. 6 - Exemplos de alguns casos com trovoadas e suas respectivas posições (ponto) em relação ao Cavado em Altos Níveis (linha tracejada) apresentando-se na (a) Vanguarda do Cavado e Retaguarda da Crista, (b) Vanguarda do Cavado, (c) Retaguarda do Cavado e Vanguarda da Crista, (d) Retaguarda do Cavado, (e) Eixo do Cavado. 


\section{Sistemas de circulação fechada}

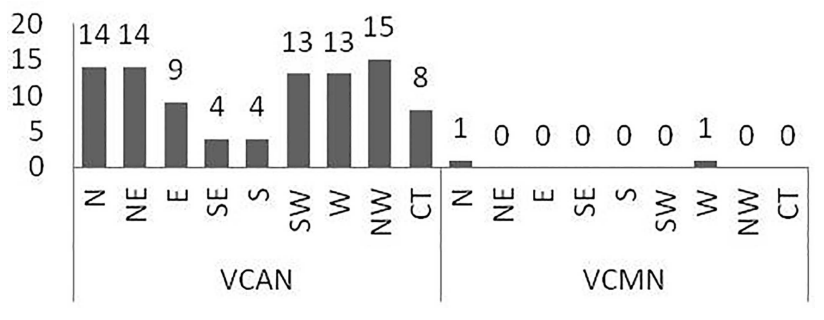

Fig. 7 - Número de eventos com trovoadas referentes ao posicionamento de seus sistemas sinóticos com circulação fechada.

Leste, Centro, Sudeste e Sul apontando 09, 08, 04 e 04 casos, respectivamente.

Para o VCMN foi identificado apenas 02 casos, no geral, sendo observada a trovoada somente a Norte e Oeste, ambos apresentando 01 caso.

Para melhor visualização foi elaborado esquemas sobre o posicionamento do fenômeno adverso em relação aos dois sistemas sinóticos mais presentes em dias com trovoadas (Fig. 8). Vale lembrar que os valores indicados na Figura 8b são a soma das posições para o Cavado em Altos Níveis.

Os movimentos verticais dependem de vários fatores e possuem grande variedade no mesmo sistema bárico (Bluestein, 1992; 1993). Nas correntes de oeste para leste, os movimentos verticais geralmente agem em regiões de vanguarda (leste) e retaguarda (oeste) do cavado, respectivamente (Djuric, 1994). Para o VCAN e Cavado em Altos Níveis, em torno deste vórtice, os movimentos verticais ascendentes predominam na periferia deste sistema (Kousky; Gan, 1981). Além disso, os movimentos verticais dependem muito da Corrente de Jato (CJ) atuante na região, onde os movimentos ascendentes são mais intensos na entrada e lado quente da CJ (Djuric, 1994; Bluestein, 1992; 1993). O aquecimento da superfície também influencia fortemente na formação de instabilidade e de movimentos verticais, os quais são um conjunto destes processos e, conseqüentemente, possuem papel principal na formação de trovoadas locais.

De acordo com dados climatológicos, na região de estudo, o centro do VCAN e eixo do Cavado em Altos

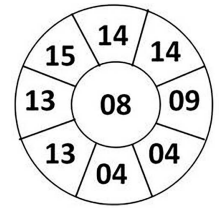

(a)

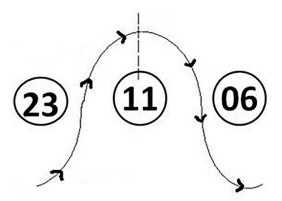

(b)
Fig. 8 - Posicionamento das trovoadas em relação aos seguintes sistemas sinóticos: (a) VCAN e (b) Cavado em Altos Níveis.
Níveis se localizam sobre o Oceano Atlântico. Por isso, a parte oeste e norte do Cavado e VCAN ficam sobre o continente, ou seja, sobre a região mais aquecida em baixos níveis, influenciando na formação de instabilidade.

\subsection{Análise termodinâmica}

Foi feito um estudo dos índices de instabilidade no intuito de avaliar a instabilidade termodinâmica do ambiente para cada sistema sinótico, através dos seguintes índices de instabilidade CAPE e LI. A Tabela 5 mostra médias de todos os eventos separados por seu sistema sinótico.

De acordo com a tabela houve certa instabilidade com a presença de Cavado em Altos Níveis, VCAN e ZCIT, indicando médias acima de $1300 \mathrm{~J} \cdot \mathrm{kg}^{-1}$. Seguindo o mesmo raciocínio, o índice LI também apresentou valores menores que $-4,5$.

Trovoadas ocasionadas por Zona Frontal mostraram instabilidade moderada, com valor médio de $1030,8 \mathrm{~J} \cdot \mathrm{kg}^{-1}$. O índice LI, por sua vez, também apresentou valor médio de $-3,8$. Já o VCMN obteve valores de índices com média de $409,5 \mathrm{~J} \cdot \mathrm{kg}^{-1}$ para CAPE e -2,0 para o LI.

\section{Conclusões}

Para o estudo de trovoadas no Estado de Alagoas foram registrados 212 casos no período de 1998 a 2012, sendo o ano de 2008 com maior número de eventos e o ano de 2012 com pouco registro do fenômeno. Os resultados também mostraram que as trovoadas atuaram com mais frequência dentre os meses de Janeiro, Fevereiro e Março.

Os sistemas sinóticos encontrados nos dias com trovoadas foram Cavado em Altos Níveis, VCAN, VCMN, ZCIT e Zona Frontal. O sistema que mais atuou foi VCAN com 94 casos, em seguida Cavado em Altos Níveis com 40 casos. Por outro lado, VCMN obteve sua menor ocorrência apresentando somente 02 casos. Se comparar com estudos feitos anteriormente (Brito, 2011), houve um aprimoramento no que diz respeito às análises feitas na parte sinótica, ou seja, nos estudos anteriores foram encontrados apenas a presença dos ventos Alísios com curvatura

Tabela 5 - Valores médios dos índices de instabilidade CAPE e LI, para cada sistema sinótico estudado.

\begin{tabular}{lcc}
\hline \multicolumn{2}{c}{ Médias } & \\
\hline Sistemas sinóticos & CAPE & LI \\
\hline VCAN & 1319,6 & $-4,5$ \\
CAVADO EM A.N. & 1374,4 & $-4,6$ \\
ZONA FRONTAL & 1030,8 & $-3,8$ \\
ZCIT & 1479,1 & $-5,2$ \\
VCMN & 409,5 & $-2,0$ \\
\hline
\end{tabular}


ciclônica (em baixos níveis), VCAN e Corrente de Jato no Nordeste brasileiro (CJNEB - em altos níveis) e nenhum sistema em médios níveis.

Para análise do posicionamento das trovoadas em relação ao sistema sinótico ocorrente, os resultados indicaram que, para o Cavado em Altos Níveis, as trovoadas ocorreram com maior frequência na região da retaguarda com 23 casos. No VCAN, as trovoadas estiveram bem distribuídas, incidindo mais à NW, $\mathrm{N}$ e $\mathrm{NE}$ do mesmo. Para o VCMN (com apenas 02 casos), foi verificado somente à $\mathrm{W}$ e $\mathrm{N}$.

Os Índices de Instabilidade analisados para cada sistema sinótico mostraram que os sistemas que apresentaram maiores médias de instabilidade foram VCAN, ZCIT e Cavados em Altos Níveis.

\section{Agradecimentos}

O presente trabalho foi realizado com o apoio do Conselho Nacional de Desenvolvimento Científico e Tecnológico (CNPq).

\section{Referências}

BARROS, A.H.C.; FILHO, J.C.deA.; SILVA, A.B. da; SANTIAGO, G.A.C.F. Climatologia do Estado de Alagoas. Boletim de Pesquisa e Desenvolvimento. 2012, Recife/ PE. EMBRAPA. ISSN 1678-0892. Disponível em: http:// ainfo.cnptia.embrapa.br/digital/bitstream/item/103956/1/ BPD-211-Climatologia-Alagoas.pdf.

BLUESTEIN, H.B. Principles of Kinematics and Dynamics. In: Synoptic - dynamic meteorology in midlatitudes. Oxford: Oxford University Press, 1992. v. 1, p. 431.

BLUESTEIN, H.B. Observations and theory of weather systems. In: Synoptic - dynamic meteorology in midlatitudes. Oxford: Oxford University Press, 1993. v. 2, p. 595.

BRITO, B.M.; LEVIT V.; FEDOROVA, N.; MOLION, L.C.B.; TENÓRIO, R.S. et al. Análise do comportamento das trovoadas no estado de Alagoas, previsão à curto prazo. Revista Brasileira de Meteorologia, v. 26, n. 2, p. 243-256, 2011.

CARDOSO, N.K.R. Relações entre índices de instabilidade e as chuvas ocorridas em Porto Alegre, RS, de 1990 a 2005. Dissertação (Mestrado - Meteorologia). Universidade Federal de Pelotas. Pelotas, RS. 2007.

CAVALCANTI, I.F.deA.; FERREIRA, N.J.; SILVA, M.G.A.J. da; DIAS, M.A.FdaS. Tempo e Clima no Brasil. Oficina de Textos. São Paulo - SP. 2009.

CORDEIRO, E.S. Análise termodinâmica dos casos com trovoadas para os anos de 2008, 2009 e 2010 em Maceió, Alagoas. Trabalho de Conclusão de Curso (Graduação Meteorologia). Universidade Federal de Alagoas. Maceió, AL. 2011.

DJURIC, D. Weather Analysis. New Jersey: Prentice Hall, 1994, p. 304.

DUARTE, L.A.; WEYKAMP, F.V.E.; GOMES, R.G. Estudo da instabilidade atmosférica em um sistema convectivo de mesoescala. In: VI Inic Encontro de Iniciação Científica II Epg Encontro de Pós- Graduação, 2002, São José dos Campos. VI Inic II Epg. São José dos Campos, 2002. v. 1. p. 446-447.

FEDOROVA, N.; LEVIT, V.; CAMPOS, A.M.V. Brazilian Northeast jet stream: Association with synoptic scale systems. Meteorological Applications. DOI: 10.1002/ met.1693. 2017a

FEDOROVA, N.; LEVIT, V.; CAMPOS, A.M.V. Brazilian Northeast jet stream: frequency, Wind speed and direction. Meteorological Applications, DOI: 10.1002/ met.1688. 2017b.

FEDOROVA, N. Meteorologia sinótica. Pelotas: Ed. Universitária da Universidade Federal de Pelotas, 1999. v. 1.

FEDOROVA, N. Meteorologia sinótica. Pelotas: Ed. Universitária da Universidade Federal de Pelotas, 2001. v. 2.

FEDOROVA, N.; GEMIACKI, L.; CARVALHO, L.C.; LEVIT, V.; RODRIGUES, L.R.L.; COSTA, S.B. Frontal Zone on the North-East of Brazil. In: International Conference on Southern Hemisphere Meteorology and Oceanography (ICSHMO). Foz do Iguaçu. Proceedings. São José dos Campos: INPE, p. 1765-1768, CD-ROM, 2006.

FEDOROVA, N.; LEVIT, V.; CRUZ, C.Dda. On frontal zone analysis in the tropical region of the Northeast Brazil. Pure and Applied Geophysics, 2015. DOI 10.1007/s00024015-1166-y. ISSN: 0033-4553.

FEDOROVA, N.; LEVIT, V.; SILVA, T.G. Vórtice Ciclônico de Médios Níveis está influenciando no tempo de Alagoas? In: Atmosfera e Sociedade: Meio Ambiente e Elementos Meteorológicos, Cap. 09, Volume IV, Edufal, Maceió - Al, 139-149, 2015. ISBN 978-85-7177-919-8; CDU 551.5.504.

FEDOROVA, N.; SANTOS, D.M.B.; SEGUNDO, M.M.L.; LEVIT, V. Middle Tropospheric Cyclonic Vortex in Northeastern Brazil and the Tropical Atlantic. Pure and Applied Geophysics, 2016.

FEDOROVA, N.; SILVA, T.G. Vórtice ciclônico de médios níveis e sua influência no tempo do nordeste brasileiro. In: Meteorologia em tópicos, Cap. 3, Volume III, UFPel Pelotas, 133-178, 2016. ISBN 978-85-68891-02-5.

FELICIO, R.A. Trovoadas. (Desenvolvimento de material didático - Climatologia). Disponível em: http://www.geografia. fflch.usp.br/graduacao/apoio/Apoio/Apoio_Elisa/flg 0253/ filespdf/TrovoadasAtlasFigsCl-I.PDF,. 2005.

GALWAY, J.G. The lifted index as a predictor of latent instability. Bull. American Meteorological Society. v. 43, p. 528-529, 1956.

HALLACK, R.; PEREIRA-FILHO, A.J. Análise de desempenho de índices de instabilidade atmosférica na previsão de fenômenos convectivos de mesoescala na região metropolitana de São Paulo entre 28 de janeiro e 04 de fevereiro de 2004. In: Revista Brasileira de Meteorologia (RBMet). 2012, v. 27, n. 2, 173-206.

KÖPPEN, W. Das geographisca system der klimate. Gebr, Borntraeger, 1936. p. 1- 44.

KOUSKY, V.E.; GAN, M.L. Upper tropospheric cyclonic vortices in the subtropical South Atlantic. Tellus, Munksgaard, Copenhagen, v. 33, p. 538-551, Nov. 1981.

LEVIT, V.; CORDEIRO, E.S.; CAVALCANTE, L.C.V. Método de previsão de trovoadas no Nordeste Brasileiro. In: 
Meteorologia em tópicos, Cap. 2, Volume III, UFPel Pelotas, 79-131, 2016. ISBN 978-85-68891-02-5.

LOUREIRO, R.S. Análise de uma linha de instabilidade costeira na região leste do estado do Pará. Dissertação (Mestrado - Meteorologia). Faculdade de Meteorologia, Universidade Federal de Pelotas, Pelotas, 2005.

RODRIGUES, L.R.L.; FEDOROVA, N.; LEVIT, V. Trovoadas na costa leste do Nordeste do Brasil associadas à interação entre ondas nos ventos de leste e VCAN. In: CONGRESSO BRASILEIRO DE METEOROLOGIA (CBMET), 2008, São Paulo. Anais. São Paulo: SBMET, 2008.

SANTOS, D.M.B. Vórtices ciclônicos de médios níveis VCMN: uma análise de frequência e estrutura. Dissertação (Mestrado) - Universidade Federal de Alagoas UFAL. 2012.b.

SILVA, L. A influência do vórtice ciclônico de altos níveis (VCAN) sobre a precipitação do Nordeste do Brasil (NEB) e as características associadas. Dissertação (Mestrado em Meteorologia) - Instituto Nacional de Pesquisas Espaciais (INPE), São José dos Campos, 2005. p. 109

SILVA, B.F.P. Sistemas sinóticos associados às precipitações intensas no Estado de Alagoas. Revista Brasileira de
Meteorologia, São José dos Campos - SP, v. 26, n. 3. 2011. p. 323-338.

SIMPSON, R.H., Frank, N., Shideler, D. and Johnson, H.M. Atlantic Tropical Disturbances. Monthly Weather Review, Vol. 96, No. 4, 251-259, 1968.

VIANELLO L.R.; ALVES A.R. Meteorologia Básica e Aplicações. Viçosa (MG): Editora UFV. p. 367, 2000.

XAVIER, T.M.B.S.; Xavier, A.F.S.; Dias, P.L.S.; e Dias, M.A.F. S. A Zona de Convergência Intertropical e suas relações com a chuva do Ceará (1964-98). 2000. RBMet 15, 2743.

\section{Sítios da Internet}

Departamento de Controle do Espaço Aéreo, disponível em: http://www.redemet.aer.mil.br/?i=produtos\&p=plotagemmetar.

NCEP/NCAR, disponível em: http://www.ncep.noaa.gov.

Tropical Rainfall Measuring Mission, disponível em: http:// trmm.gsfc.nasa.gov.

This is an Open Access article distributed under the terms of the Creative Commons Attribution Non-Commercial License which permits unrestricted non-commercial use, distribution, and reproduction in any medium provided the original work is properly cited. 